\title{
Control Adaptativo de Velocidad usando el Filtro de Kalman en un Motor de Inducción
}

\author{
Santiago Sánchez ${ }^{1}$ \\ Eduardo Giraldo ${ }^{2}$
}

\section{Resumen}

En este trabajo se presenta un sistema de control que es aplicado cuando no se tiene información detallada de parámetros del sistema. Así, resulta útil emplear un modelo aproximado de la planta; esta aproximación se obtiene mediante la estimación basada en las señales de entrada y salida. La etapa de estimación es desarrollada generalmente para sistemas determinísticos evitando las incertidumbres y errores presentes debido al acondicionamiento de equipos. Se describe el cálculo en línea de parámetros para sistemas que emplean una valoración del error presente en los modelos, la sintonización adaptativa de un controlador proporcional integral y derivativo utilizando el filtro de Kalman para el control de velocidad de un motor de inducción. También, se describe el método de control en lazo cerrado de voltaje-frecuencia y la efectividad del algoritmo de control se presenta con la aplicación sobre la máquina asíncrona de corriente alterna.

\section{Palabras clave}

Control adaptativo, estimación, filtro de Kalman, motor de inducción.

1 Centro de Investigación, INSTITUTO TECNOLÓGICO METROPOLITANO, santiagosanchez@itm.edu.co

2 Programa de Ingeniería Eléctrica, Universidad Tecnológica de Pereira, egiraldos@utp.edu.co

Fecha de recepción: 05 de Abril de 2010

Fecha de aceptación: 22 de Octubre de 2010 


\section{Abstract}

This work presents a control system used when the detailed parameter information of a plant in not available. Therefore, is useful to design a controller with an approximated model of the system, this is reachable through the use of parameter estimation based on the input and output signals. The estimation procedure is commonly developed with deterministic algorithms, avoiding the presented uncertainties and errors added to the system by the conditioning equipment. In this work we present an on line parameter computation, over systems that employs an error assumption in their model, the adaptive tuning of a PID controller using the Kalman filter in order to control the speed on an induction motor. Also, the control by the voltage-frequency in closed loop and the effectiveness of the control algorithm by the test developed on the asynchronous machine are described.

\section{Keywords}

Adaptive control, estimation, induction machine, Kalman filter. 


\section{INTRODUCCIÓN}

El control adaptativo es ampliamente usado dado su robustez en sistemas que presenten una variación en los parámetros sea por efectos de temperatura, afectados en forma externa, el modelo tenga una elevada complejidad o sea no lineal y requiera de trabajo alrededor de un punto de equilibrio. Estos efectos de cambio hacen que el diseño de un controlador por algún otro método que no considere las variaciones disminuya el desempeño; en la industria y a través de los años uno de los controladores más utilizados es el proporcional, integral y derivativo (PID) por su sencilla sintonización y aplicación, en este trabajo se presenta el desarrollo de una técnica de control adaptativa que utiliza como método de estimación el filtro de Kalman; como controlador el regulador PID y como sistema a controlar un motor de inducción jaula de ardilla (MI). El motor de inducción se ha convertido en la máquina de corriente alterna de uso en la industria y en aplicaciones innovadoras las cuales buscan reducir la contaminación; entre estas están las aplicaciones en el diseño de automóviles híbridos y los motores de los trenes eléctricos, su utilidad radica en su estructura robusta, elevada habilidad para operar en ambientes pesados.

La selección del motor de inducción como máquina industrial es debido a que su relación peso por potencia es menor que una máquina de corriente continua, en las máquinas A.C. se presentan los fenómenos de variación en los parámetros dado a calentamientos en el devanado lo que causa incertidumbre y reduce la exactitud del controlador, se tienen dificultades con la fricción desconocida y la perturbación en el par de carga variable; adicionalmente el modelo es no linealy presenta un fuerte acople entre el flujo y el torque. Leonhard (2001) presenta una descripción del modelo y de los métodos de control del MI.

Chiasson $(1998,2005)$ y Sánchez \& Giraldo (2009) presentan la técnica de control velocidad con linealización por realimentación de las variables de estado, la cual hace necesario el conocimiento detallado de los parámetros del sistema y una etapa de sintonización de un regulador PI interno, esta técnica está sujeta a que las variaciones de los parámetros afecten el desempeño del 
controlador, otra técnica de control no lineal es presentada en (Mezouar et al., 2007) donde se emplean modos deslizantes adaptativos; sin embargo este método requiere de gran complejidad en el diseño e implementación. La técnica de control por campo orientado (Bose, 2005) genera una etapa de diseño de alta complejidad, en (Rehman \& Nounou, 2007) se emplea un controlador difuso adaptativo para máquinas de corriente alterna que tiene el entrenamiento con la técnica de gradiente descendente, además lo hace lento en la convergencia de parámetros estimados y computo. Las técnicas de control adaptativo presentan una ventaja sobre el motor y otros sistemas, además hace más sencillo el diseño e implementación del controlador. En (Ustun \& Dermitas, 2009) se realiza un controlador con un sistema ANFIS de inteligencia artificial que emula el motor, entrenamiento del regulador PI sintonizado por algoritmos genéticos y control del inversor por voltaje/frecuencia, pero el empleo de tablas de datos de entradas y salidas al sistema hace que el diseño requiera de un tiempo de entrenamiento largo; además de emplear un sistema fuera de línea.

En este trabajo se controla la velocidad del eje del motor, en la sección 2 se describe el filtro de Kalman en general, la siguiente sección presenta la descripción de un modelo discreto autorregresivo de entrada auxiliar; el cual es empleado para tener en cuenta el ruido registrado en la adquisición causado por la vibración del sensor de velocidad acoplado a la máquina de inducción, la sección 4 presenta el uso del filtro de Kalman sobre el problema de estimación de parámetros. La sección 5 describe el diseño de controladores PID mediante la reubicación de polos diseñado por Astrom y las dos últimas secciones describen el modelo de control por voltaje/frecuencia propuesto en Bose (2005) con la adición del control adaptativo, las pruebas y resultados que tienen en cuenta las incertidumbres del sistema.

\section{MODELO MOTOR DE INDUCCIÓN}

El modelo en los ejes d-q, el cual es el equivalente en dos fases del sistema trifásico del motor de inducción se presenta en (1)-(6). 


$$
\begin{aligned}
& \frac{d \theta}{d t}=\omega \\
& \frac{d \omega}{d t}=\mu \varphi_{d} i_{q}-\frac{f}{J} \omega-\frac{T_{L}}{J} \\
& \frac{d \varphi_{d}}{d t}=-\eta \varphi_{d}+\eta L_{m} i_{d} \\
& \frac{d i_{d}}{d t}=-\gamma i_{d}+\left(\frac{\eta L_{m}}{\sigma L_{r} L_{s}}\right) \varphi_{d}+n_{p} \omega i_{q}+\eta L_{m} \frac{i_{q}^{2}}{\varphi_{d}}+\frac{u_{d}}{\sigma L_{s}} \\
& \frac{d i_{q}}{d t}=-\gamma i_{q}+\left(\frac{\eta L_{m}}{\sigma L_{r} L_{s}}\right) n_{p} \omega \varphi_{d}-n_{p} \omega i_{d}-\eta L_{m} \frac{i_{q} i_{d}}{\varphi_{d}}+\frac{u_{q}}{\sigma L_{s}} \\
& \frac{d \rho}{d t}=n_{p} \omega+\eta L_{m} \frac{i_{q}}{\varphi_{d}}
\end{aligned}
$$

donde $u_{d}, u_{q}$, son los voltajes del estator en el eje directo $\mathrm{y}$ cuadratura respectivamente, $i_{d}, i_{q}$, las corrientes de estator, $\varphi_{d}$ la magnitud del flujo del rotor, $\rho$ el ángulo del flujo del rotor, $\omega$ es la velocidad del rotor, $R_{r}, R_{s}$ son las resistencias del rotor y estator respectivamente, $L_{s} L_{r}$ y $L_{m}$ son las inductancias de estator, rotor y de acoplamiento, $J$ es el coeficiente de amortiguamiento, $f$ es el coeficiente de viscosidad, $n_{p}$ el número de polos y $T_{L}$ es el par de carga (Chiasson, 2005; Fitzgerald et al., 2003). Los parámetros se describen en (7).

$\eta \stackrel{\Delta R_{r}}{L_{r}} ; \beta=\frac{\Delta L_{m}}{\sigma L_{r} L_{s}} ; \mu=\frac{\Delta n_{p} L_{m}}{J L_{r}} ; \gamma=\frac{\Delta L_{m}^{2} R_{r}}{\sigma L_{r}^{2} L_{s}}+\frac{R_{r}}{\sigma L_{s}}$

\section{FILTRO DE KALMAN}

El filtro de Kalman ofrece una forma de estimar el estado $x_{k}$ de un modelo en un sistema estocástico lineal de dimensión finita dado por (8), (9) y (10) donde $v_{1 k}$ y $v_{2 k}$ representan entradas de ruido blanco. Si se remueven las señales de ruido $v_{1 k} \mathrm{y} v_{2 k}$ el modelo se reduce a un sistema de espacio de estado determinístico 
(Goodwin \& Sin, 1984; Haynki, 1996; Katayama \& Sugimoto, 1997; Gelb et al. 1980).

La estimación del proceso se puede ver como una forma de control por realimentación: el filtro estima el estado en algún tiempo y luego obtiene la realimentación en la forma de las medidas (más ruido). Las ecuaciones para el filtro de Kalman se presentan en dos grupos: actualización de tiempo y actualización de la medida.

La secuencia $\left\{v_{1 k}\right\}$ se llama ruido del proceso y $\left\{v_{2 k}\right\}$ es el ruido de la medida u observación, estos son procesos estacionarios de ruido blanco con media cero y no correlacionados.

$$
\begin{aligned}
& x_{k+1}=A x_{k}+B u_{k}+v_{1 k} \\
& y_{k}=C x_{k}+v_{2 k} ; \quad k \geq k_{0}
\end{aligned}
$$

donde $x_{k 0}$ es una variable aleatoria de media $\bar{x}_{0}$ y covarianza $\Sigma_{0} \mathrm{y}$

$E\left\{\left[\begin{array}{c}v_{1 k} \\ v_{2 k}\end{array}\right]\left[\begin{array}{cc}v_{1 k}^{T} & v_{2 k}^{T}\end{array}\right]\right\}=\left(\begin{array}{cc}Q & S \\ S^{T} & R\end{array}\right)$

$Q \geq 0, \quad R>0$

$E$ es el primer momento estadístico. Las matrices de covarianza $Q$ y $R$ se asumen constantes y la matriz de covarianza $S$ puede ser cero.

El filtro se basa en las dos interpretaciones siguientes: Si el ruido es Gaussiano, el filtro presenta la estimación del estado de mínima varianza. Si se remueve la asunción, el filtro obtiene la estimación del estado de mínima varianza lineal.

El teorema de Kalman se basa en la obtención de una ecuación que estime el estado posterior $\hat{x}_{k+1}$ como una combinación lineal del estado anterior $\hat{x}_{k}$, una ponderación de la diferencia entre la medida actual $y_{k}$ y una predicción de la medida $C \hat{x}_{k}$ y la relación de la entrada de control $B u_{k}$, la descripción anterior se presenta en (11) y (12). 


$$
\begin{aligned}
& \hat{x}_{k+1}=A \hat{x}_{k}+K_{k}\left[y_{k}-C \hat{x}_{k}\right]+B u_{k} \\
& \hat{x}_{k 0}=\bar{x}_{0}
\end{aligned}
$$

donde $K_{k}$ es la ganancia de Kalman dada por (13).

$$
K_{k}=\left[A \Sigma_{k} C^{T}+S\right]\left[C \Sigma_{k} C^{T}+R\right]^{-1}
$$

Se define el error de la estimación del estado en (14).

$e=\widehat{x}_{k}-x_{k}$

$\Sigma_{k}$ es la covarianza del error de estado dadas las observaciones $\left\{y_{k}\right\}$, su definición se presenta en (15).

$\Sigma_{k} \stackrel{\Delta}{=} E\left\{\left[\hat{x}_{k}-x_{k}\right]\left[\hat{x}_{k}-x_{k}\right]^{T} \mid y_{k-1}, \ldots, y_{k 0}\right\}$

$\Sigma_{k}$ satisface la ecuación de diferencia de Riccati (16) y(17).

$\Sigma_{k}=A \Sigma_{k} A^{T}+Q-K_{k}\left[C \Sigma_{k} C^{T}+R\right] K_{k}^{T}$

$\Sigma_{k 0}=\Sigma_{0}$

\section{MODELO ARX}

Modelo autorregresivo de entrada auxiliar. Se llama de esta manera para hacer diferencia con el modelo AR, dado que este utiliza el ruido blanco, con media cero y varianza constante no correlacionado con la entrada o la salida (Goodwin \& Sin, 1984; Ljung, 1999).

Este modelo se representa en ecuaciones de diferencia de la forma presente en (18).

$$
y_{k}+a_{1} y_{k-1}+\ldots+a_{m} y_{k-m}=b_{0} u_{k}+b_{1} u_{k-1}+\ldots+b_{m} u_{k-m}+e_{k}
$$


Donde $m$ es el orden del modelo, $y$ la salida del sistema, $u$ la señal de control de la planta y $e$ el error. El modelo de la ecuación 18 puede representarse con (19) para el caso de estimación.

$y_{k}=\phi^{T}{ }_{k} \theta_{k}+e_{k} \quad$ para $\quad k \geq 0$

Donde $\theta_{k}$ es la matriz de parámetros de $m \times p$ y $\phi_{k}$ es un vector de $p \times 1$ que contiene los valores pasados de la salida y la entrada.

\section{ESTIMACIÓN DE PARÁMETROS USANDO KALMAN}

El modelo del proceso se describe como se muestra en (20), se toma $\theta_{k}$ como el vector de estados definido en el filtro.

$y_{k}=\phi_{k}^{T} \theta_{k}+e_{k}$

Se puede escribir una ecuación del estado como en (21), la cual toma como base (8).

$\theta_{k+1}=\theta_{k}+w_{k}$

Para este caso al aplicar el filtro de Kalman al sistema anterior, $B=0, A=I$ y $C=\phi_{k}^{T}$ (Ljung, 1999; Tarvainen et al., 2004, 2006; Georgiadis et al., 2005).Se asume que el estado inicial no está correlacionado con los errores y con varianza finita, los dos grupos de ecuaciones del filtro se presentan desde (22) hasta (27). Siendo (22) y (23) las actualizaciones de tiempo y desde (24) a (27) la actualización de la medida.

$$
\begin{aligned}
& \hat{\theta}_{k \mid k-1}=\hat{\theta}_{k-1} \\
& \Sigma_{k \mid k-1}=\Sigma_{k-1}+C_{w k-1} \\
& K_{k}=\Sigma_{k \mid k-1} \varphi\left[\varphi^{T} \Sigma_{k \mid k-1} \varphi+C_{e k}\right]^{-1}
\end{aligned}
$$


$\Sigma_{k}=\left(I-K_{k} \varphi^{T}\right) \Sigma_{k \mid k-1}$

$\xi_{k}=y_{k}-\varphi^{T} \hat{\theta}_{k \mid k-1}$

$\hat{\theta}_{k}=\hat{\theta}_{k \mid k-1}+K_{k} \xi_{k}$

donde $\hat{\theta}_{k \mid k-1}$ es la estimación media para $\theta_{k}$ dadas las observaciones $y_{1}, \ldots, y_{k-1}$ y $K_{k}$ es la mencionada matriz de ganancia de Kalman.

\section{DISEÑO DE CONTROLADORES PID USANDO REUBICACIÓN DE POLOS}

El modelo de control general puede ser descrito en la Fig. 1.

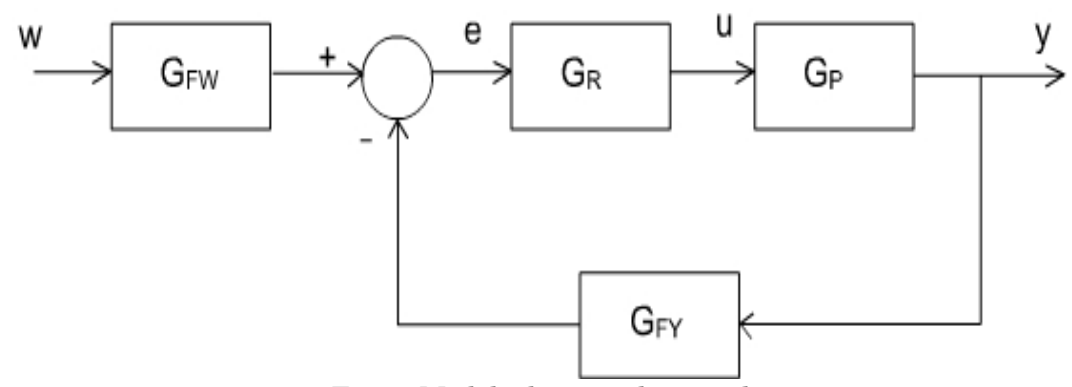

Fig. 1. Modelo de control general

De la Fig. 1. GFW: es la ganancia del filtro de entrada, GR: la función de transferencia que define al controlador, GP: la función de transferencia que define la planta y $G F Y$ : función de transferencia del lazo de realimentación, para el diseño del controlador requerido $G F W$ y $G F Y$ son iguales a la unidad, $G R$ es el controlador proporcional integral y derivativo. Conceptualmente el controlador PID es un controlador de parámetros lineales optimizado, con estructura fija dada por la función de transferencia de (28). 
$G_{R}(z)=\frac{q_{0}+q_{1} z^{-1}+q_{2} z^{-2}}{1-z^{-1}}$

donde $q_{0}, q_{1}$ y $q_{2}$ son los parámetros del PID. Astrom en 1980 diseñó un controlador para un proceso de orden dos y un modelo de referencia de orden dos (Iserman et al., 1992; Iserman, 1980). Para obtener el PID extendido de (29), con una selección apropiada de parámetros del controlador se puede obtener el sistema proporcional, proporcional integral y el proporcional integral derivativo.

$G_{R}(z)=\frac{q_{0}+q_{1} z^{-1}+q_{2} z^{-2}}{\left(1-z^{-1}\right)\left(1+\gamma_{1} z^{1}\right)}$

El PID puede ser diseñado con la técnica de reubicación de polos, la ecuación característica en lazo cerrado (LC) de (30).

$$
\begin{aligned}
E c\left(z^{-1}\right) & =\left(1-z^{-1}\right)\left(1+a_{1} z^{-1}+\ldots+a_{m} z^{-m}\right)+\left(q_{0}+q_{1} z^{-1}+q_{2} z^{-2}\right)\left(b_{1} z^{-1}+\ldots+b_{m} z^{-m}\right) \\
& =1+\alpha_{1} z^{-1}+\ldots+\alpha_{m+2} z^{-m+2}
\end{aligned}
$$

Las raíces de la ecuación son los polos del sistema en lazo cerrado y quedan determinados por (31).

$$
\left(z-z_{\alpha 1}\right) \ldots\left(z-z_{\alpha(m+2)}\right)=0
$$

\section{CONTROL DEL INVERSOR POR VOLTAJE/FRECUENCIA}

Este método de control del inversor es el más difundido en la industria por su fácil implementación y bajo costo de cómputo. En aplicaciones que requieran variación de velocidad es necesario que el voltaje sea proporcional a la frecuencia tal que se tenga una operación de par constante. En principio no se requiere de un lazo de realimentación, pero para garantizar un mejor desempeño se puede implementar control en lazo cerrado. La señal de referencia para el control del dispositivo electrónico es la velocidad angular 
eléctrica $\left(\omega_{e}\right)$, el voltaje requerido es proporcional a $\omega_{e}$; siendo necesario solo el ajuste de la constate de proporcionalidad.

\section{PRUEBAS Y RESULTADOS}

La Fig. 2 presenta el sistema de pruebas empleado, se encuentra el motor de inducción jaula de ardilla, la tarjeta de adquisición de datos, el sensor de velocidad y el inversor. Las características del motor empleado son: $240 \mathrm{~V}, 1,5 \mathrm{~A}, 1 / 3 \mathrm{HP}, 3$ fases, 1725 RPM, 4 polos, se realizó para las pruebas en tiempo real un programa en el lenguaje $\mathrm{C}++$ y la adquisición y salida de datos con una tarjeta NI PCI MIO 6070E, de 1,25 MS/s. El método de control del accionamiento es por voltaje-frecuencia.

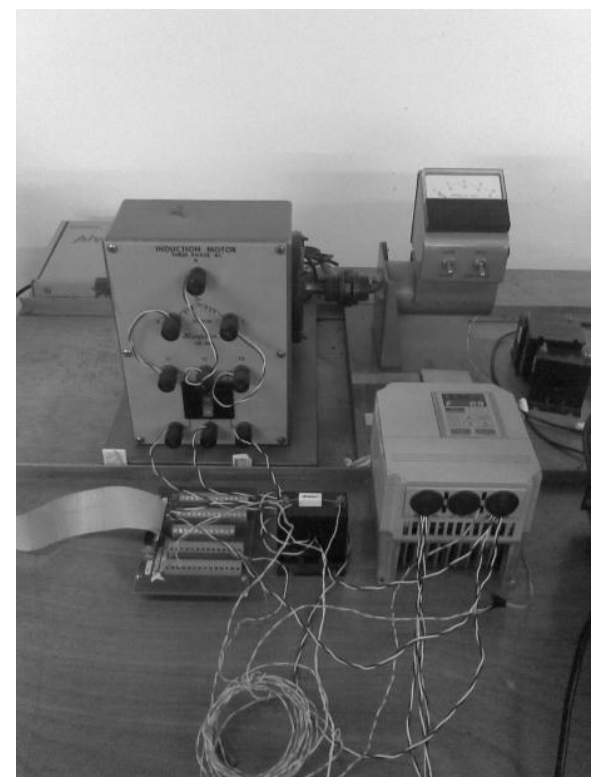

Fig. 2. Sistema de pruebas empleado

Para la implementación del sistema adaptativo se utiliza el sistema en lazo cerrado con el cual se mejora la respuesta dinámica, se pretende que la velocidad se mantenga constante 
siguiendo una referencia sin importar el par de carga. En el control de velocidad, se ubican los polos como se presenta en (32); para utilizar el seguimiento tipo dead-beat los polos son localizados en el origen.

$$
E c\left(z^{-1}\right)=1+\alpha_{1} z^{-1}+\ldots+\alpha_{m+2} z^{-m+2}=1
$$

El modelo de control PID adaptativo actualiza los valores de los parámetros en cada iteración y genera la señal de control requerida para lograr el seguimiento necesario, el PID adaptativo utilizado es el realizado por Astrom el cual incorpora un polo más en la función de transferencia, de esta forma se resuelve el problema de los parámetros para un modelo de segundo orden.

Los resultados del sistema de control adaptativo implementado sobre el motor de inducción, se analizan con base en las Fig. 3-5 en esta prueba se realiza solo estimación hasta los $22 \mathrm{~s}$; esto se puede observar en la Fig. 3; donde la señal de velocidad de la planta aparece con línea continua y la estimada por el sistema con línea discontinua, representando el proceso de reconstrucción de la señal de velocidad basado en los parámetros estimados por el filtro de Kalman.

El error de estimación se presenta en la Fig. 4, en la etapa de estimación se tiene error cero a partir de la tercer muestra; debido a la espera para almacenar los datos pasados de entrada y salida del algoritmo, la variación de los parámetros encontrados durante esta etapa de estimación se presentan en la Fig. 5. A partir de los $22 s$ se habilita el controlador PID adaptativo y se continua realizando la estimación de parámetros de la señal de salida basado en los parámetros estimados.

De la Fig. 6, la señal de control varía entre sus límites inferior y superior [0-10] $V$ respectivamente, esta es la entrada al amplificador de voltaje y representa el voltaje aplicado al circuito del estator; en la gráfica el sistema está en lazo abierto hasta los $22 s$ donde se varía con un escalón para la etapa de estimación, en adelante el sistema es cambiado al lazo cerrado y la señal de control es obtenida de forma automática por el control PID adaptativo. 


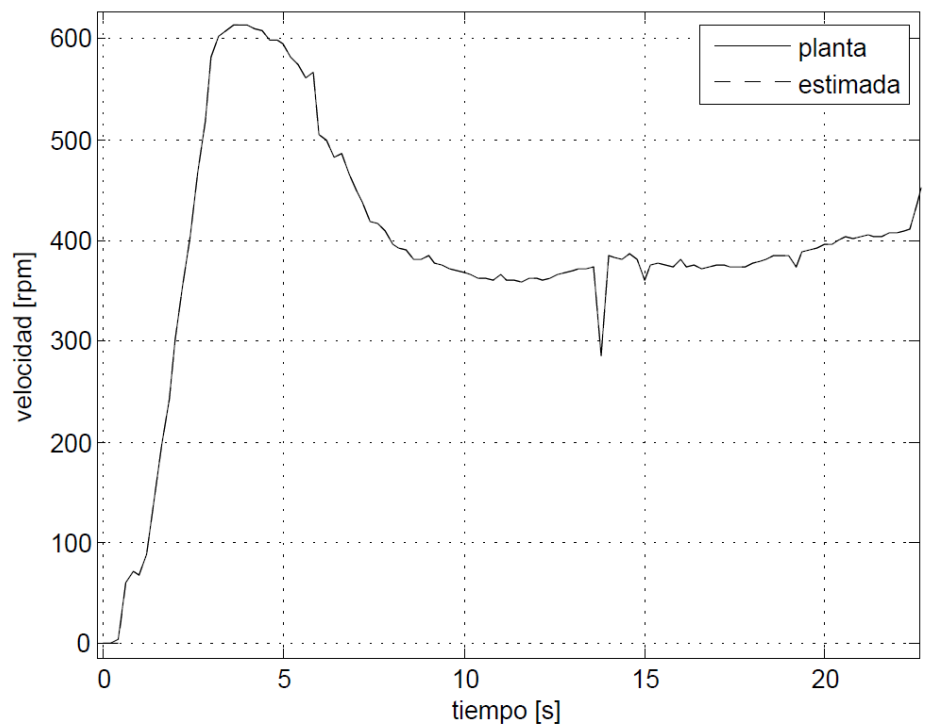

Fig. 3. Estimación de la señal de salida

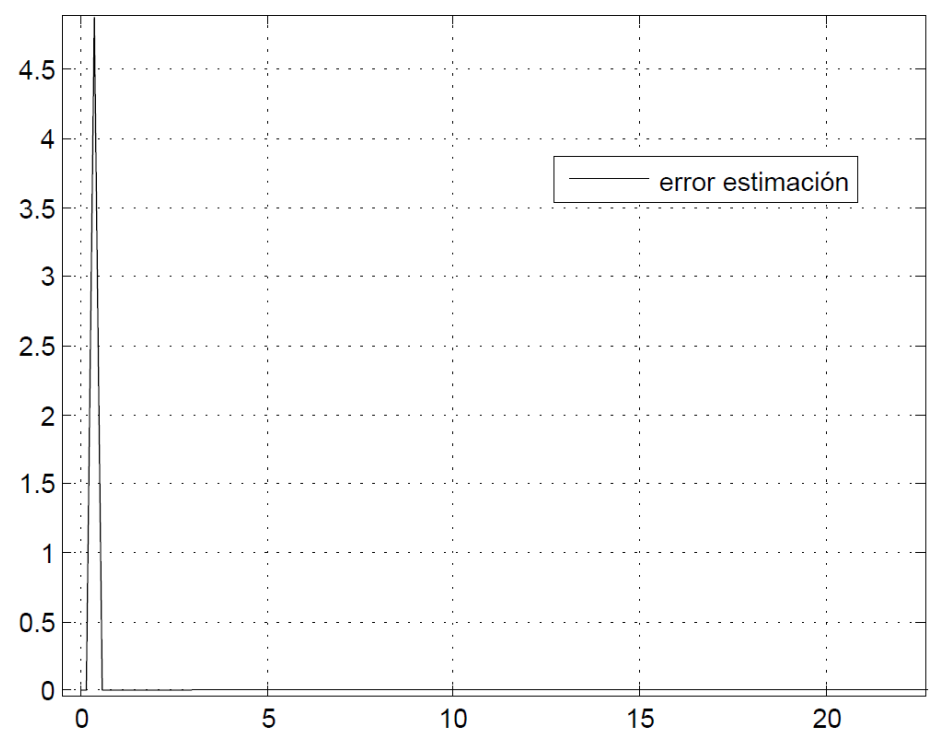

Fig. 4. Error de estimación de la señal de salida 


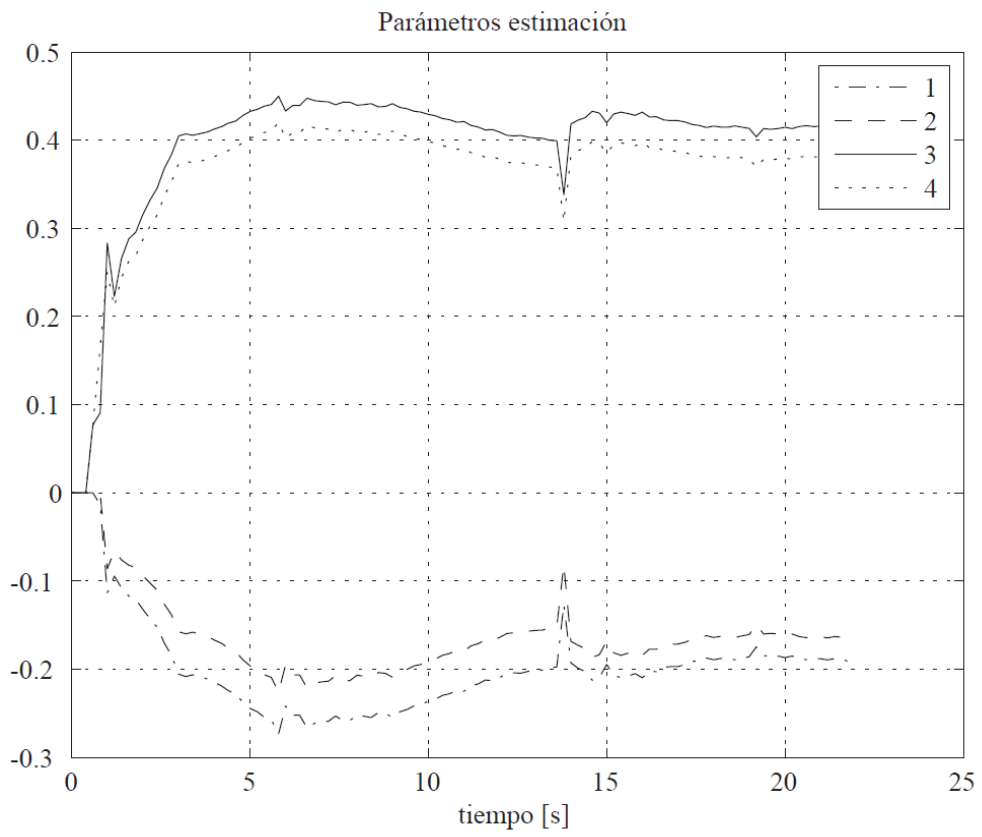

Fig. 5. Parámetros en la etapa de estimación

En la Fig. 7 se presenta el momento del cambio de estimación en lazo abierto a control en lazo cerrado, se observa el sobrepaso de la velocidad del motor respecto a la señal de referencia al inicio de la etapa de control, luego la referencia es variada en donde los cambios de velocidad del rotor son suaves y alcanza la velocidad deseada de forma rápida; al principio la velocidad es cambiada de $400 \mathrm{rpm}$ a $600 \mathrm{rpm}$ con el voltaje de entrada variando de $2,4 \mathrm{~V}$ a $4,8 \mathrm{~V}$ aproximadamente, luego se incrementa a $1000 \mathrm{rpm}$ produciendo otro aumento en la señal de control, en esta etapa de control los parámetros se mantienen estables lo que se puede observar de la Fig. 8; así se generen cambios bruscos en la velocidad deseada. Durante la etapa de control se continúa realizando la estimación de la señal de velocidad, en la cual se mantiene un error cercano a cero (ver Fig. 9). 


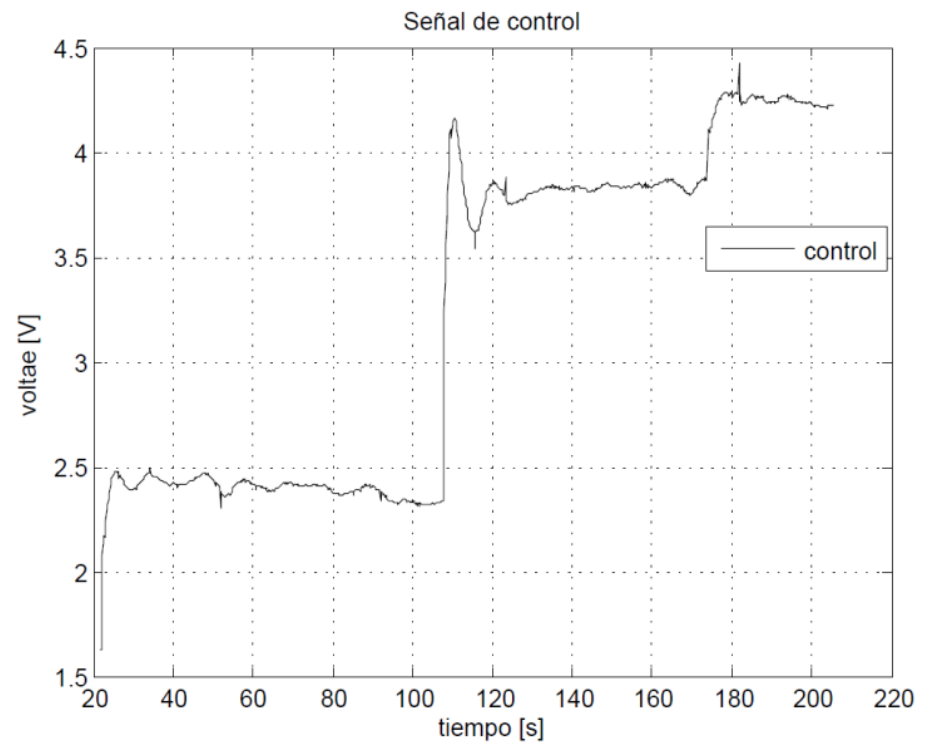

Fig. 6. Señal de control

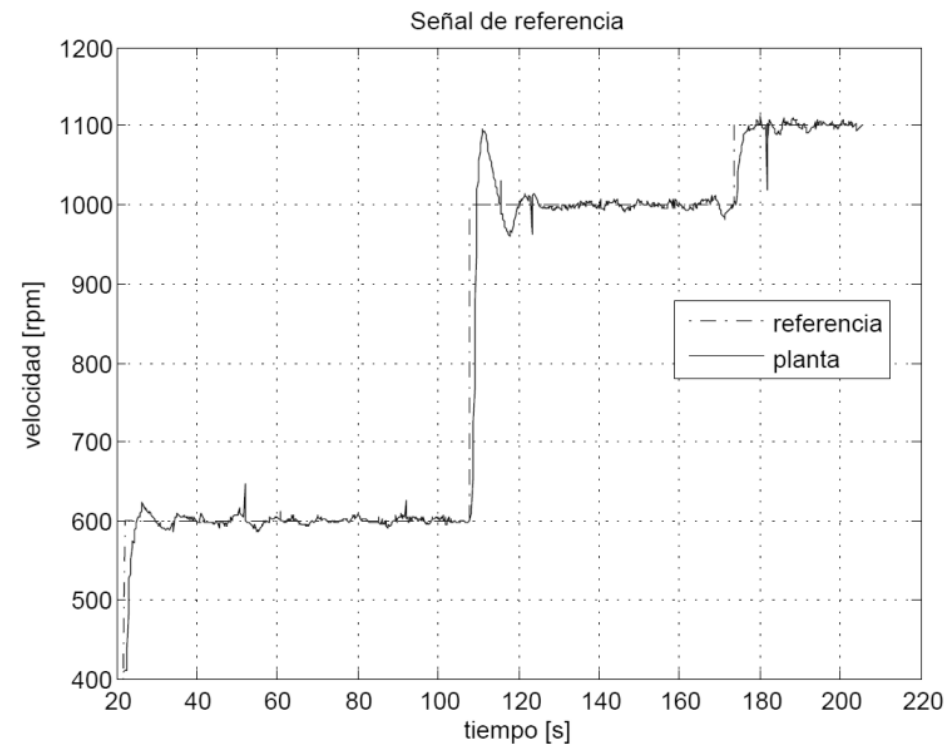

Fig. 7. Etapa de seguimiento 


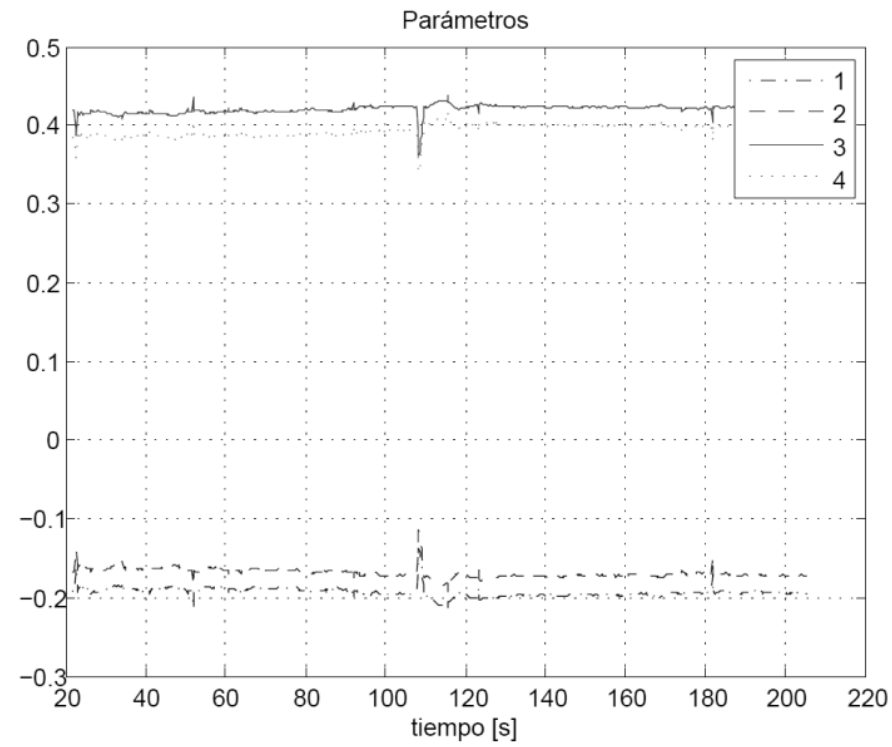

Fig. 8. Variación de los parámetros durante la etapa de control

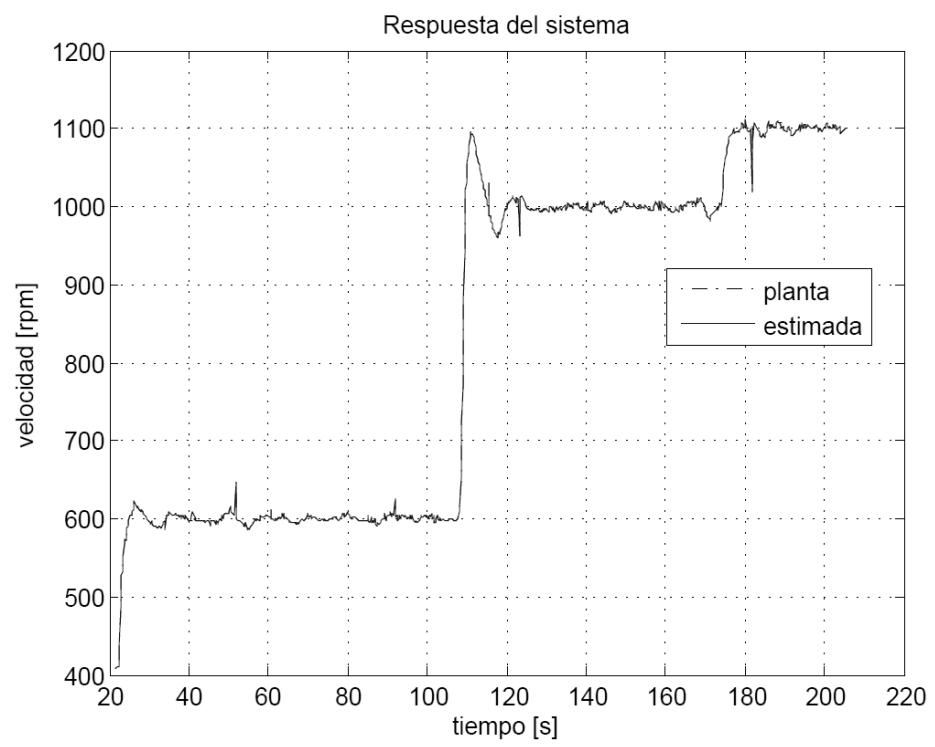

Fig. 9. Estimación de la velocidad durante el control 
En la Tabla 1 se presenta una comparación de las estrategias de control por V/f; la primera empleada en este trabajo el controlador adaptativo con filtro de Kalman para la sintonización de regulador PI (CAK) y el trabajo de Ustun que emplea un modelo neuro-difuso del motor y sintonización del regulador mediante algoritmos genéticos (CFGA) con tabla de datos de entrada-salida(Ustun \& Dermitas, 2009).Se observa que para simplificar el diseño el controlador adaptativo solo requiere de la ubicación de polos, con los cuales se tendrá la dinámica deseada de la planta.

Tabla 1. Comparación de las técnicas de control

\begin{tabular}{ccccc}
\hline & $\begin{array}{c}\text { Facilidad de } \\
\text { implementación }\end{array}$ & $\begin{array}{c}\text { Sintonización } \\
\text { en línea }\end{array}$ & $\begin{array}{c}\text { Control no } \\
\text { lineal }\end{array}$ & $\begin{array}{c}\text { Problemas ante } \\
\text { variación } \\
\text { paramétrica }\end{array}$ \\
\hline CAK & $\mathrm{Si}$ & $\mathrm{Si}$ & $\mathrm{Si}$ & Baja \\
CFGA & $\mathrm{No}$ & $\mathrm{No}$ & $\mathrm{Si}$ & Media \\
\hline
\end{tabular}

\section{CONCLUSIONES}

Se ha implementado una técnica de control adaptativa utilizando el filtro de Kalman para la manipulación de la velocidad en un motor de inducción. El controlador PID extendido adaptativo ha sido diseñado usando la técnica de reubicación de polos para controlar la respuesta dinámica y la de estado estable del motor. Los valores de la señal de voltaje se mantienen dentro de los límites permitidos por el sistema (entrada análoga del variador V/f).Se utilizó una aproximación de la planta reduciendo el modelo a uno de orden dos para generar los parámetros del controlador PID obteniendo resultados de bajo error y control en un amplio rango de velocidades. La aplicación del sistema de control es presentada en forma práctica obteniendo unos resultados satisfactorios. 


\section{REFERENCIAS}

Bose, B.K., (2005); Modern Power Electronics and AC drives, 56-74, 339345, Prentice Hall. New York.

Chiasson, J., (1998); A new approach dynamic feedback linearization control of an induction motor. IEEE Transaction on Automatic Control, Vol. 43, 391-397.

Chiasson, J., (2005); Modeling and High Performance Control of Electric Machines, 511-518, IEEE Press Series on Power Engineering, WileyInterscience, New York.

Fitzgeral, A.E, Kingsley, Jr. C., \& Umans, S.D., (2003); Electric Machinery, 6th edition, 664-667, United States.

Gelb, A., Kasper, J.F., Nash, R.A. \& Price, C.F., (1980); Applied Optimal Estimation, 102-136, MIT Press, USA.

Georgiadis, S., Tarvainen, M.P., \& Karjalainen, P.A., (2005); Single-trial Dynamical Estimation of Event Related Potentials: A Kalman FilterBased Approach, IEEE Transactions on Biomedical Engineering, 52(8), 1397-1406.

Goodwin, G.C. \& Sin, K. S., (1984); Adaptive Filtering Prediction and Control, 330-340, Prentice Hall, Englewood Cliffs, New Jersey.

Haynki, S., (1996); Adaptive Filter Theory, 302-334, Prentice Halland Systems Sciences Series, 3rd edition, New Jersey.

Iserman, R., (1980); Digital Control Systems, Fundamentals, deterministic control, 1, 138-143, Spreinger-Verlag, Berlin.

Iserman, R., Lachmann, K.H., \& Matko, D., (1992); Adaptive Control Systems, 100-103, Prentice Hall. New York.

Katayama, T. \& Sugimoto, S., (1997); Statistical Methods in Control and Signal Processing, 1-8, 74-78, Marcel Dekker, New York.

Leonhard, W., (2001); Control of Electrical Drives, 164-175, 252-260, 3rd edition. Springer, New York.

Ljung, L., (1999); System Identification, Theory for the user, 361-370, 2nd edition, Prentice Hall, Sweden.

Mezouar, A., Fellah, M.K., \& Hadjeri, S., (2007); Adaptive sliding mode observer for induction motor using two-time-scale approach. Electric power system research, 77, 604-618. 
Rehman, H. \& Nounou, H.N., (2007); Application of fuzzy control to ac machines, Applied soft computing, 7, 899-907.

Sánchez, S. \& Giraldo, E., (2009); Sistemas de Control en modelos de máquinas eléctricas, 93-95, Publicaciones Universidad Tecnológica de Pereira. Colombia.

Ustun, S.V. \& Dermitas, M., (2009), Modeling and control of V/f controlled induction motor using genetic-ANFIS algorithm, Energy Conversion and Management, 50, 786-791.

Tarvainen, M.P., Georgiadis, S. \& Karjalainen, P.A., (2006), Time-Varying Analysis of Heart Rate Variability with Kalman Smoother Algorithm, Procedings of the 2005 IEEE Engineering and Medicine and Biology 27th Annual Conference, Shangai, China, 2718-2721.

Tarvainen, M.P., Hiltunen, J.K., Ranta-aho, P.O. \& Karjalainen, P.A., (2004), Estimation of Nonstationary EEG with Kalman Smoother Approach: an Application to Event-Related Synchronization, IEEE Transaction on Biomedical Engineering, 51(3), 516-524. 
